\title{
Del determinismo a la agencia: relatos de estudiantes egresados de enseñanza media técnico profesional que acceden a la universidad
}

\author{
From Determinism to Agency: Narratives of \\ Secondary Technical-Vocational Education Graduates \\ Who Access University
}

\section{Claudia Mónica Concha Saldías, Gerardo Ignacio Sánchez Sánchez, y Caroll Alejandra Schilling Lara}

Universidad Católica del Maule, Chile.

\begin{abstract}
Resumen
Este artículo tiene como propósito aportar a la compresión de la experiencia educativa de egresados de la enseńanza media técnico profesional que cursan estudios en tres universidades regionales del Consejo de Rectores de Chile. A través de sus relatos, se muestra cómo, a pesar de las trayectorias de pobreza que vivencian, sortean el determinismo que pesa sobre su origen social y logran ingresar a la universidad. Metodológicamente, se recurrió a un diseńo cualitativo: mediante 32 entrevistas episódicas, se indaga en la experiencia de la educación técnico profesional e integración académica y social en la universidad. Los resultados revelan la presencia de una narrativa colectiva, respecto de la precariedad de la formación recibida durante la enseñanza media y la presión que ejerce el habitus familiar y escolar por la incorporación temprana al mundo laboral. Superando el determinismo, los jóvenes exhiben una capacidad de agencia al desplegar diversas estrategias para su ingreso y permanencia en la universidad.
\end{abstract}

Palabras claves: educación técnico profesional, desigualdad educativa, tracking curricular, habitus, integración social y académica.

Correspondencia a:

Claudia Mónica Concha Saldías.

Universidad católica del Maule, Facultad de Ciencias Sociales y Económicas, Escuela de Sociología, Avenida San Miguel 3605, Talca, Chile.

mconcha@ucm.cl

Se agradece a la Comisión Nacional de Acreditación que financió la investigación, así como a las tres universidades regionales adscritas al Consejo de Rectores de Chile que voluntariamente participaron en el estudio, y especialmente a los estudiantes que de manera generosa compartieron sus trayectorias educativas, abriendo la reflexión respecto de las desigualdades educativas y los desafíos en la formación profesional.

(C) 2021 PEL, http://www.pensamientoeducativo.org - http://www.pel.cl 


\section{Abstract}

The objective of this paper is to contribute to the understanding of the educational experience of graduates of technical-vocational secondary schools who study at three regional universities that belong to the Council of Rectors of Chilean Universities. Their narratives show how, despite the trajectories of poverty they experience, they overcome the determinism that hangs over their social origin and manage to enter university. Methodologically, the study used a qualitative design: through 32 episodic interviews, we analyzed the experience of technicalvocational education and academic and social integration at university. The results reveal the presence of a collective narrative regarding the precariousness of the education received during high school and the pressure exerted by the family and school habitus for early entry into the labor market. Overcoming this determinism, the young people demonstrate a capacity for agency by deploying various strategies to enter and remain at university.

Keywords: Vocational education, educational inequality, curricular tracking, habitus, social and academic integration.

\section{Introducción}

En las últimas décadas, Chile evidencia un crecimiento económico relativamente estable, aunque una persistente desigualdad (Organización para la Cooperación y el Desarrollo Económicos [OCDE], 2015). Esta se expresa cotidianamente en malestar y sentimientos de injusticia, al percibir que los accesos a ciertos bienes socialmente valorados se distribuyen injustamente, minando lazos y vínculos sociales, generando odio y situaciones de violencia, que impiden la integración y cohesión social (Programa de las Naciones Unidas para el Desarrollo [PNUD], 2017). Frente a estas desigualdades, las sociedades modernas utilizan diversos mecanismos para acortar las brechas, siendo uno de los más relevantes, y con mayor reconocimiento social, la educación.

Para las visiones liberales, la educación sería el mecanismo que está llamado por excelencia a promover la movilidad social, y jugaría un rol importante en la modificación de las estructuras sociales (Mauna, 2013). Sin embargo, en contraste con esta visión liberal de la educación como el gran igualador, están los teóricos de la reproducción, quienes conciben a la educación como un mecanismo de reproducción de la ideología dominante, de la distribución del conocimiento y de las habilidades necesarias para mantener la división social del trabajo. Para los teóricos de la resistencia, en tanto, existe la capacidad de agencia del sujeto que se opone a los modelos de la cultura dominante o la reproducción social que imponen las instituciones educativas (Hirsch \& Rio, 2015).

Nuestro país, ha privilegiado en los últimos 30 años a la educación como mecanismo de movilidad social y medio principal para disminuir la vulnerabilidad social (PNUD, 2017). Para ello, ha implementado una serie de políticas orientadas a mejorar la calidad y la equidad de la educación mediante cambios curriculares, inversión en infraestructura, programas de acceso inclusivo, aumento de financiamiento para familias vulnerables y gratuidad, entre otros, permitiendo el acceso a la educación superior. Pese a esto, existe una marcada estratificación social del sistema educativo, reflejado en un desigual acceso a establecimientos de calidad, escenario que profundiza la segregación social y las condiciones de desigualdad, concentrando en el sector municipal a los más pobres de la población (Larrañaga, Cabezas, y Dusaillant, 2013). A esto se añade que el último ciclo de enseñanza media se organiza en dos modalidades curriculares: la científico-humanista (en adelante, EMCH) y la técnico-profesional (en adelante, EMTP). Esta última concentra el 37\% de la matrícula de enseñanza media y $79 \%$ de sus egresados pertenece al nivel socioeconómico bajo, y 21\%, al medio bajo (Ministerio de Educación [MINEDUC], 2020). 
Este artículo tiene como propósito aportar a la comprensión de la experiencia educativa de jóvenes egresados de la EMTP, que cursan estudios en tres universidades regionales del Consejo de Rectores de Chile. Por tanto, se busca indagar en el análisis que hacen los jóvenes de su trayectoria educativa y social, sus subjetividades y las propias constricciones que impone la formación técnico-profesional, así como la capacidad de agencia que movilizan mediante diversas acciones y estrategias para sortear los procesos de inclusión/exclusión social en la universidad.

La literatura denomina tracking curricular a la diferenciación entre formación EMTP y EMCH, noción que explica cómo un sistema educativo separa tipos de currículo y enseñanza, exponiendo a los estudiantes a diferentes entornos institucionales, oportunidades de aprendizaje, grupos de pares, y expectativas sociales (Sevilla, 2017; Shavit \& Müller, 2000). Investigaciones nacionales (Agencia de Calidad de la Educación, 2016; Arias, Farías, González-Velosa, Huneeus, y Rucci, 2015; Larrañaga, Cabezas, y Dussaillant, 2013; Bassi y Urzúa, 2010) muestran que los estudiantes que eligen la EMTP presentan resultados de aprendizajes inferiores en el sistema de medición de la calidad de los aprendizajes, SIMCE 2015, obteniendo 30 puntos menos en pruebas de lectura y ciencias sociales, y 50 puntos menos en matemáticas; en tanto, en la Prueba de Selección Universitaria, PSU 2015, obtienen 106 puntos menos en promedio que los estudiantes EMCH.

Más allá de los resultados, está claro que el discurso construido en torno a la educación superior como mecanismo de movilidad social es una razón significativa para que jóvenes egresados de la EMTP movilicen sus esfuerzos hacia el nivel superior (Arias et al., 2015). Esto se constata en el aumento del porcentaje de egresados de EMTP que ingresaron a la educación superior entre los años 2008 y 2017, el que pasó de 21\% a 44\% (Ministerio de Educación, [MINEDUC], 2020). Esta situación podría explicarse por políticas de acceso inclusivo y de financiamiento, como la gratuidad.

Sin embargo, existen evidencias nacionales (Farías y Carrasco, 2012; Larrañaga, Cabezas, y Dussaillant, 2014) que muestran que los egresados de EMTP que ingresan a la educación superior, obtienen en promedio 30 puntos menos en la prueba de selección universitaria (PSU), limitando la elección de carreras y centros educativos de calidad. A esto se añade que la tasa de deserción se duplica en los estudiantes de la modalidad EMTP. En tanto, estudios internacionales muestran que los estudiantes que optan por la modalidad técnico-profesional reciben una formación con un currículo menos exigente y con proyección exclusiva al mercado laboral, profesores con bajas expectativas, y pares de menor nivel socioeconómico (Arum \& Shavit, 1995).

A los elementos estructurales de la desigualdad educativa se agregan evidencias en el ámbito cualitativo (Canales y De los Ríos, 2009; Concha, 2009; Castillo y Cabezas, 2010; Mejía-Pérez y Worthman, 2017) que describen cómo los jóvenes en situación de vulnerabilidad experimentan la integración social y académica. Tal como lo advierten Canales, Opazo y Camps (2016):

A la universalización de la educación media siguió un colectivo fuertemente orientado hacia caminos de educación terciaria. En el paso de salir del cuarto, la sociedad prometía que todos iban a ser estudiantes de tercer ciclo, evitando o conjurando así el fantasma del regreso al trabajo simple de la mayoría de sus familias y vecinos (pp.102-103).

\section{Discusión teórico conceptual}

El tracking curricular refiere a la diferenciación del currículum que adoptan los sistemas educativos, organizando trayectorias de formación, al menos una académica y otra técnico-vocacional (Sevilla, 2017). La evidencia muestra (Ainsworth \& Roscigno, 2005; Reay, David, \& Ball, 2001; McDonough, 1988) que el acceso a una u otra trayectoria formativa estaría mediado por elementos estructurales y simbólicos, entre ellos las características sociodemográficas, las creencias, y las expectativas de los estudiantes y de sus familias. 
En sociedades desiguales y altamente segregadas como la chilena, la elección de la modalidad técnico-profesional estaría fuertemente relacionada con el menor nivel socioeconómico y educativo de las familias y la mayor oferta de este tipo de establecimientos en la comuna de residencia del estudiante (Larrańaga et al., 2013; Raczynski, Hernández, Rejevic, y Roco, 2011, de Iruarrizaga, 2009).

Estudios nacionales e internacionales describen ampliamente la relación entre tracking curricular y segregación social. Sevilla y Polesel (2020) analizan el vínculo entre el primero y las desigualdades sociales en el sistema educativo chileno. Específicamente, revelan que la elección de la modalidad educativa EMTP está influenciada por el bajo nivel socioeconómico de los estudiantes, quienes presentan mayores brechas en el acceso a la educación superior en relación con sus pares que cursan la modalidad EMCH.

En un sistema segregado, el perfil del estudiante tiende a ser más homogéneo al interior de las escuelas: los alumnos de origen social similar que se concentran en escuelas de su zona residencial reproducen las condiciones del contexto social que habitan. Así, las áreas desfavorecidas ven afectada la calidad de los servicios en términos de la infraestructura disponible, el prestigio, el tamaño de las escuelas y los profesores que trabajan en ella (Ong \& Rickles, 2004; Jenkins, Micklewright, \& Schnepf, 2008). A esto se añade el llamado efecto pares (Bellei, 2013; Valenzuela, Bellei, y De los Ríos, 2009; Orfield, 2001), que dice relación con la interacción entre estudiantes en el contexto escolar y cómo este puede agregar ventajas o desventajas a sus aprendizajes. Al respecto, Dumay y Dupriez (2008), Trevińo et al. (2010), y Zimmer y Toma (2000) advierten que la concentración de población vulnerable en las escuelas tiene efectos negativos en los aprendizajes y logros de los estudiantes.

A todos estos elementos se añaden las expectativas y valoración de la educación de las familias de contextos desaventajados respecto del futuro académico de sus hijos, el que estaría ligado preferentemente al trabajo, lo que podría explicar que la elección formativa esté más vinculada a la formación EMTP, y menos orientada a los estudios superiores (Catalán, 2016). En ese sentido, Dávila, Ghiardo y Medrano (2005) advierten que “(...) los grupos con menor escolaridad presentan trayectorias históricamente más ligadas al trabajo que a la educación, situándolos en lógicas de carácter práctico, con salidas más directas hacia el mundo del trabajo que a la continuación de estudios" (p.168)

En este escenario, estudiantes que provienen de contextos de pobreza experimentan al menos dos tensiones que afectan su aspiración educativa futura: el origen social de las familias (habitus de origen) y la institución escolar (habitus institucional). Existe una extensa literatura que explicaría la influencia de las familias en las trayectorias educativas. Para Bourdieu (1980), el habitus es un "sistemas de disposiciones duraderas y transponibles, estructuras estructuradas predispuestas a funcionar como estructuras estructurantes...” (p.88). Esto se refiere a la configuración de principios que generan prácticas y representaciones que son adoptadas por los grupos sociales sin resistencia.

Bourdieu y Passeron (1995) explican que

(...) la reproducción de las relaciones de clase son el resultado de una acción pedagógica, que no parte de una tabula rasa, sino que se ejerce sobre sujetos que recibieron de sus familias o de las acciones pedagógicas procedentes (la llamada 'educación primera'). Cada acción pedagógica tiene, pues, eficacia diferenciada en función de las diferentes caracterizaciones culturales preexistentes de los sujetos y que son de naturaleza social. La escuela, al sancionar estas diferencias como si fueran puramente escolares, contribuye al mismo tiempo a reproducir la estratificación social y a legitimarla (p.17).

Las estrategias de reproducción constituyen un sistema que permite activar un conjunto de prácticas mediante las cuales los agentes, de manera consciente o inconsciente, conservan o aumentan su capital, lo que permite mantener o mejorar su posición en la estructura social. Ańaden los autores que la estrategia más usada por las familias en la sociedad moderna, para asegurar la posición social de los herederos, es la obtención de credenciales educativas. 
Desde esta perspectiva, el establecimiento escolar donde se haya estudiado, las profesiones y universidades por las que se opte, el tipo de trabajo que se realiza, y las redes sociales de las cuales se dispone son todos factores que permiten comprender las condiciones objetivas y subjetivas de situarse en el espacio social (Dávila et al., 2005).

Siguiendo estas ideas, Crozier, Reay, Clayton, Colliander y Grinstead (2008) proponen la noción de habitus institucional, concebida como conjunto de predisposiciones, esquemas de percepción y expectativas a través de las cuales se organiza un centro educativo. Siguiendo a estos autores,

(...) constituyen el capital encarnado e incorporado en la colectividad de los estudiantes, en la cultura, en su forma de vestir, el comportamiento y las actitudes, en particular, sus actitudes hacia el aprendizaje y su grado de confianza y el derecho en relación con el conocimiento académico (p.109).

Para Reay (1998), toda organización social posee un habitus institucional identificable, fuertemente relacionado con el contexto cultural y socioeconómico en el que está inserta, el que determinaría las percepciones y expectativas que el sujeto construye en relación con los puntos de vista de los amigos y maestros y mediante las experiencias de aprendizaje. Por lo tanto, la opción de continuar estudios en la educación superior estará subordinada en gran parte por el efecto escolar, o el llamado habitus institucional, como lo advierten Reay et al., (2001).

Si bien el habitus es un sistema de disposiciones que genera esquemas de comportamiento, no es una estructura inalterable que el sujeto interioriza sin posibilidad de cambio; todo lo contrario, impulsado por el interés personal o colectivo, realiza un conjunto de experiencias que se van grabando, almacenando, y que recogen la influencia de diversos ambientes que sucesivamente se han encontrado a lo largo de una vida. La agencia del sujeto sobre estas estructuras permitirá que "se encuentra con un mundo social del que es producto, es como un 'pez en el agua": no siente el peso del agua y da por sentado el mundo sobre sí mismo" (Bourdieu \& Wacquant, 1992, p.127).

Aisenson (2002) advierte que, si bien los jóvenes son afectados por el contexto en que viven, son activos en la significación de los hechos que les suceden, capaces de anticipar y responder de manera personal a los cambios externos y conflictos internos de su transición educativa. La escuela y las relaciones sociales que los sujetos establecen en su interior permitirían el despliegue de acciones sobre las estructuras represivas, las que variarán de escuela a escuela y de barrio a barrio (Giroux, 1985), abriendo espacio para la convivencia entre la autoproducción y la reproducción.

También es posible que los jóvenes aprovechen las oportunidades que el propio sistema ofrece y que les permiten romper con las trayectorias lineales y los determinismos; ejemplo de esto son las políticas públicas, que recogen el anhelo del acceso y financiamiento. Si bien los discursos dominantes se construyen en términos de las capacidades académicas -meritocracia-, premiando con el acceso a quienes han tenido un logro educativo destacable, los jóvenes construyen su participación sobre la lógica de la capacidad académica de la que disponen para sortear la permanencia en la universidad en términos de integración social y académica, y los riesgos económicos y sociales que implica el fracaso (Archer \& Hutchings, 2000).

\section{Metodología}

La investigación se enmarcó en un paradigma constructivista, perspectiva que da cabida a formas de investigación más complejas, que asumen que las realidades son aprehendidas bajo la forma de múltiples construcciones mentales y que su origen se fundamenta en la experiencia social de los individuos y colectivos (Guba y Lincoln, 2002). Bajo este paradigma, se optó por la estrategia metodológica cualitativa dado su carácter flexible y abierto, y porque este tipo de diseño permite abordar el estudio de problemáticas en la educación superior, profundizando en la comprensión de las subjetividades e indagando en la reflexión personal y social del estudiante; así como en su relación con el 
mundo escolar y académico (Ashworth, 2000). La técnica de producción de información fue la entrevista episódica, que permite recoger la experiencia de las personas y generar conocimiento de tipo narrativo vinculado a episodios específicos, así como conocimiento semántico, esto es, reflexiones, explicaciones y relaciones más abstractas, que se organizan a partir de tales experiencias (Flick, 2004). El guion de la entrevista abordó los tópicos (i) experiencia en la educación técnico-profesional, en términos de elección y valoración; (ii) adaptación a la vida universitaria, en los ámbitos sociales y académicos, y (iii) costos y beneficios asociados a la permanencia en la universidad.

Las entrevistas se realizaron en tres universidades regionales del Consejo de Rectores de Chile, y la técnica de muestreo fue intencionada. A partir de la base de datos proporcionada por cada institución, se procedió a invitar a los participantes bajo los siguientes criterios de selección: i) estudiante egresado de EMTP, matriculado en una de las tres universidades en el periodo 2015-2017; ii) pertenecer a carreras con alta y baja presencia de egresados de EMTP, y iii) voluntariedad para participar en la investigación. El número de casos de la muestra operó bajo el principio de redundancia o saturación, entendido como el agotamiento de la información o efectos de sentido no conocidos previamente (Canales, 2006). Se realizaron 32 entrevistas aplicadas durante el primer semestre de 2019, que tuvieron una duración de entre 50 a 90 minutos-y se efectuaron en dependencias de las casas de estudio.

Tabla 1

Muestra seleccionada del estudio

\begin{tabular}{|c|c|c|c|c|c|c|}
\hline \multirow[t]{2}{*}{ Carrera } & \multicolumn{2}{|c|}{ Género } & \multicolumn{2}{|c|}{$\begin{array}{l}\text { Procedencia } \\
\text { geográfica }\end{array}$} & \multicolumn{2}{|c|}{$\begin{array}{c}\text { Tipo de establecimiento } \\
\text { de origen }\end{array}$} \\
\hline & Femenino & Masculino & Urbano & Rural & Municipal & Subvencionado \\
\hline Ingeniería comercial & 1 & 1 & 1 & 1 & 1 & 1 \\
\hline Ingeniería civil & & 1 & 1 & & 1 & \\
\hline Ingeniería civil industrial & & 2 & 1 & 1 & 1 & 1 \\
\hline Ingeniería informática & & 2 & 2 & & 1 & 1 \\
\hline Ingeniería en construcción & & 1 & & 1 & 1 & \\
\hline Geología & 1 & & & 1 & 1 & \\
\hline Enfermería & 3 & & 3 & & 3 & \\
\hline Bioquímica & 1 & & 1 & & 1 & \\
\hline Medicina veterinaria & 1 & & & 1 & 1 & \\
\hline Medicina & 1 & & & 1 & 1 & \\
\hline Biotecnología & 1 & & 1 & & 1 & \\
\hline Psicología & 1 & 2 & 3 & & 2 & 1 \\
\hline Sociología & & 3 & 3 & & 3 & \\
\hline Derecho & 1 & & 1 & & 1 & \\
\hline Educación parvularia & 3 & & 3 & & 2 & 1 \\
\hline Educación física & 3 & 1 & 2 & 2 & 4 & \\
\hline Pedagogía básica & 1 & & & 1 & 1 & \\
\hline Pedagogía en lenguaje & 1 & & & 1 & 1 & \\
\hline Total & 19 & 13 & 22 & 10 & 28 & 5 \\
\hline
\end{tabular}

Fuente: elaboración propia. 
Las entrevistas se analizaron mediante codificación abierta, para luego proceder a una codificación axial, la que "activa sistemáticamente la relación que guardan los códigos y las familias (o subcategorías y categorías, respectivamente) entre sí" (Bonilla-García y López-Suárez, 2016, p. 308). Las principales categorías que emergieron son: i) elección familiar del centro escolar; ii) experiencia formativa en el liceo, e iii) ingreso a la universidad y proceso de integración académica y social. Con este material, se discutieron los resultados y se formularon conclusiones en torno al objetivo del estudio.

\section{Resultados}

\section{a. Tener la cartita bajo la manga}

La opción de estudiar en un liceo técnico-profesional se explica desde una racionalidad práctica, que proviene de dos vertientes. Por una parte, los jóvenes, al evaluar sus condiciones socioeconómicas precarias, visualizan el área técnica como un medio que a corto plazo les permite el acceso a ingresos económicos; por otra, los padres ven en esta trayectoria formativa la seguridad de la reproducción de su prole, mediante la adquisición de credenciales que habilitarían a sus hijos e hijas en un oficio para la incorporación temprana al mundo laboral.

Mis viejos, ellos más de una vez nos conversaron de que cuando nosotros éramos más chicos, ellos nos veían trabajando no más, tener una familia, una casita. Por eso, al momento de elegir liceo, seguramente nos dijeron que la mejor opción era un liceo técnico, porque íbamos a sacar un cartón técnico que nos ayudara a encontrar trabajo (E3).

La presión de mis padres, ellos querían que igual tuviera un título técnico, decían [que era] tener la cartita bajo la manga, entonces era como tener una seguridad laboral para más adelante, en caso de que no hubiese posibilidades de ingresar a alguna universidad, instituto (E4).

Existe conciencia en los jóvenes de la precariedad socioeconómica de sus hogares y de las limitadas opciones educativas que poseen, percibiéndose así que las familias no tienen un campo real de elección. Esta experiencia es acompañada de la sensación de riesgo si optaran por la modalidad científico-humanista, debido a que los sujetos evalúan que la inversión es alta y podría no rendir beneficios a corto plazo. De esta manera, el tracking curricular de estos jóvenes respondería a la estructura de oportunidades disponible, afectando a las trayectorias formativas, a la percepción de segregación académica y a la formación de identidades subjetivas y sociales que expresan el malestar de la desigualdad (Sevilla, 2017; Shavit \& Müller, 2000). No obstante, se evidencia que existe un proceso reflexivo por parte de los jóvenes que, analizando las limitaciones del contexto de su vida cotidiana, planean alternativas para fortalecer sus capacidades y disminuir futuros riesgos.

Estudié electricidad, no me gustaba, pero lo hacía porque tenía que hacerlo, además (...) estudié en el liceo industrial porque era la mejor opción que había para mí (E7).

No sabía si iba a poder seguir en una universidad, entonces si me arriesgaba a irme a un científicohumanista podía salir sin nada, entonces en vez de arriesgarme preferí irme a un técnico-profesional, porque al menos salía con un título (E10).

En el reconocimiento de una desigualdad estructural, los jóvenes construyen expectativas educativas restringidas que, en la mayoría de los casos, no contemplan inicialmente una formación universitaria. Se añade que las familias no poseen un habitus que se vincule con el espacio social universitario y sus prácticas. Por lo tanto, las expectativas educativas se construirían sobre las experiencias previas de sobrevivencia y por la socialización en otros proyectos de vida profesional. Desde la literatura se advierte la influencia del capital cultural de la familia en las trayectorias educativas de los hijos (Ainsworth \& Roscigno, 2005). 
Nunca había pensado entrar a la universidad, siempre pensé que ir a la universidad no era tan necesario para surgir. No era tan importante, porque ninguno de mis familiares tenía grado universitario, yo pensaba 'para qué voy a ir a la universidad si no quiero ser abogado, no quiero ser médico y más encima es muy caro y tienen muchas dificultades para poder entrar’ (E7).

Mis padres no terminaron la media, llegaron a segundo medio, por ahí. Mis tíos fueron técnicos agrícolas, nosotros veíamos que les iba bien, entonces técnico era como la expectativa. Y elegí el colegio técnico profesional (E22).

\section{b. Mi liceo era un desastre...}

Los estudiantes relatan su experiencia en el liceo técnico a partir del contexto socioeconómico del establecimiento, la relación con sus profesores y compañeros, la calidad de la formación y las expectativas docentes. El colectivo de los discursos juveniles devela la situación de segregación social que se vive cotidianamente, expresada en comportamientos de oposición y resistencia al proceso de enseñanza, percibiendo la realidad escolar como caótica, incierta y poco segura.

Las relaciones sociales en los establecimientos públicos se despliegan en un ambiente de tensión entre la propuesta formativa de los docentes y la falta de interés de los estudiantes. Existe resistencia por parte de los jóvenes hacia las formas de dominación que expresa el currículo y que no representan su vida cotidiana, y menos sus intereses. Los habitus de origen se enfrentan con los habitus institucionales, afectando los aprendizajes, las interacciones sociales y la calidad de los ambientes escolares (Dumay \& Dupriez, 2008; Treviño et al., 2010; Zimmer \& Toma, 2000).

Mi liceo era un desastre, tenía una reputación cultural (...) bastante mala. La gente que salía de ahí realmente era porque no tenía otra opción en esta vida, (...) no están interesados en aprender o tienen otras prioridades, muchos de ellos son papás primerizos, a los 16, 15, entonces están preocupados de otras cosas (E11).

Los chicos del industrial son muy pesados, son muy buenos para agredir a los profesores, no físicamente, pero verbalmente, o despreciarlos, porque no les gusta la materia que hacen, entonces claro, ellos al final se aburren y tampoco ponen de su parte, entonces el estudiante y el profe deberían ir de la mano (E13).

Para los estudiantes de establecimientos subvencionados confesionales, la experiencia educativa se vive con mayor resguardo. Estos espacios se caracterizan por ser más tranquilos y familiares, dotando al estudiante de una experiencia que los protege entre iguales.

Éramos un colegió solo de mujeres, así que la relación con el sexo masculino no se daba mucho.

(...) Existía una excelente relación, nunca sufrí bullying, ni vi que hicieran. Era un ambiente súper tranquilo, me llevé muy bien con los profesores, como uno está desde chiquitito lo conocen de prácticamente toda la vida, entonces al final son como medios profesores y familia (E4).

En cuanto a la calidad de los procesos formativos, en general se califican como deficientes; se destaca que los profesores, al igual que el promedio de los estudiantes, no tienen interés en los procesos de enseñanza, y se los percibe como realizadores de una tarea o administradores. En este escenario, estos estudiantes se reconocen como aventajados en cuanto a su rendimiento académico, aunque seńalan invertir poco tiempo y esfuerzo, el que se remite a poner atención en clases, generando escasos hábitos de estudio.

Tenía mi cuaderno organizado, pero de estudiar en la casa como que no era mucho, era de poner atención en clases. Es que en el liceo no nos enseñaban a organizarnos ni nada, y como la exigencia era baja, no había necesidad de estudiar tanto (E44). 
La cantidad de ejercicios que nos hacían en el colegio eran súper pocos, los eran muy básicos, o sea, ver logaritmos, con suerte creo que vimos lo básico. Si uno comparara la matemática de enseñanza media, era como de octavo básico (E26).

Finalmente, se identifica que la opción técnica está orientada a estudiantes con trayectorias educativas débiles, con déficit académicos, pues las exigencias y expectativas del medio escolar son escasas, limitando las proyecciones educativas futuras. Para Reay y sus colegas (2001), las escuelas poseen habitus institucionales identificables, y estos se encuentran fuertemente relacionados con el contexto cultural y socioeconómico, determinando las percepciones y expectativas que el sujeto construye a lo largo del tiempo.

En el liceo, los técnicos siempre éramos como los más bajo a nivel estudiantil y académico. De hecho, tenía una profesora que siempre nos decía que nunca íbamos a ser alguien en la vida por estudiar en un técnico (E5).

Los profesores, en realidad, si bien hacían su trabajo de enseñar el tema práctico y el tema teórico, tampoco es que íbamos a ir a la universidad; en realidad, ellos se enfocaban en sí a entregar toda la información que era para desempeñarse en este trabajo (E23).

\section{c. En la universidad no todo es color de rosas.}

Para los jóvenes entrevistados, la integración en la universidad se relaciona con las posibilidades que tienen los estudiantes de vincularse a compañeros y profesores en espacios extraacadémicos, el resultado y compromiso con sus estudios, el desarrollo de habilidades socioemocionales, y la correspondencia de sus esfuerzos con las expectativas de sus profesores. Por lo tanto, la llegada a la universidad se vive con incertidumbre; la formación técnico-profesional no los habilitó en conocimientos, administración y organización del tiempo, ni en la autonomía requerida para navegar en las exigencias académicas, situación considerada como fuente importante de estrés. Se añade el sentimiento de frustración, debido a que en sus liceos eran los de mejor rendimiento, por lo tanto, sin experiencias de fracaso académico. Esto requiere tanto el despliegue de la agencia como de capacidades para avanzar en su proceso de adaptación, que aseguran su permanencia y progresión en la universidad.

En la universidad no todo es color de rosas, (...) hay cosas que a uno no le dicen, por ejemplo... cuando visité la universidad en cuarto medio, uno ve a los universitarios que lograron llegar acá, son superiores de cierta manera, pueden optar a un futuro mejor, y después uno entra en la $\mathrm{U}$, tengo que esforzarme, pero uno se da cuenta que a pesar de todo el esfuerzo que le ponga hay cosas que no resultan (E25).

Mi primer año fue horrible, no traía hábitos de estudio, para mí todo era práctico. Yo entraba a un taller, preparaba cosas, ahí me sacaban notas. Acá tuve que llegar a leer mucho, mucho... Hace mucho tiempo que no leía. Tenía buenas notas en el colegio, y en la universidad se me hizo muy complicado, reprobé ramos y nunca me había echado un curso ${ }^{1}$, nunca había tenido un promedio bajo 5.0, y aquí me he sacado un 2.0. Fue una experiencia traumante [traumatizante], la universidad (E8).

Se agrega el cuestionamiento a una educación media que no los prepara para el acceso a la universidad. Sin embargo, estos desniveles iniciales son contrarrestados a partir de disposiciones personales asociadas a responsabilidad y perseverancia, y como consecuencia, a un decidido compromiso con los estudios universitarios. Ello pone de manifiesto la presencia de un sujeto con capacidad de agencia frente a los desafíos de integración. Tal como lo

1. Chilenismo para "reprobar un curso". 
advierte Aisenson, (2002) si bien los jóvenes se ven afectados por el contexto en el que viven, son capaces de anticipar y responder de manera personal a los cambios externos y conflictos internos de su transición educativa, evidenciando un dominio de las operaciones de anticipación y planificación de la acción, más allá de los determinismos.

Los estudiantes identifican en el tracking curricular técnico profesional un factor que impide en gran medida estar en un mejor punto de partida para enfrentar las exigencias académicas universitarias. Esto se acompaña de habitus institucionales que podrían favorecer u obstaculizar la integración. Los relatos ponen de manifiesto las dificultades para lograr comunicarse con los profesores, entender el proceso de evaluación y asumir la reprobación. En definitiva, muestran las complejidades que deben sortear para hacer frente a los códigos del mundo académico.

Me eché un ramo, el profesor me decía, 'esto tú tienes que haberlo aprendido antes. Yo no te lo enseño ahora'. Lo buscaba en la oficina, nunca estaba disponible, nunca entregaba las pruebas, así como 'mira, te equivocaste en esto, esto y esto', las notas aparecían mágicamente, esa fue como una pésima experiencia que tuve (E18).

El individualismo es común en los profesores, uno les va a preguntar algo, y si él quiere te responde o si no, simplemente, 'no, tengo una reunión, me voy', o uno le manda correos y no responden, o se le hace alguna pregunta en clase y dice 'no, después te respondo' (E7).

A partir del reconocimiento de la dureza del primer ańo, y de asegurar la permanencia en la universidad, los relatos identifican la importancia del perfil de profesor de primer año, considerado un factor clave que facilitaría la integración académica. Si bien las disposiciones personales colaboran en los procesos de integración, valoran la cercanía del profesorado y las competencias pedagógicas en el proceso de formación profesional.

Los profesores de primer año no deben ser tan duros, o sea, ser exigentes a la vez, pero tener un poco más de comprensión con estos alumnos que vienen de diferentes lados, porque muchos vienen recién saliendo de la casa. Enfrentarse con profes que son demasiado 'te puede cortar cabeza', que si no estudias te va a ir mal, como ese tipo amenazas (E8).

En primer año, yo creo que incentivar un poco más a los alumnos, porque uno llega perdido, básicamente, así como al desierto. Como que te mandan a la lucha, ¿me entiende? En ese sentido, encuentro que tienen que ser más cercanos a los alumnos (E24).

Gradualmente, los estudiantes, haciendo uso de los dispositivos que provee la institución, en el encuentro con sus iguales y en el despliegue de sus propios recursos personales, logran comprender el espacio formativo y adaptarse a él. Esta experiencia que vive el estudiante, como parte de su integración social, es condicionada por el habitus institucional y, en definitiva, por el reconocimiento que la institución muestra hacia las características culturales de este tipo de estudiantes. Un aspecto clave que impacta directamente en los destinos del estudiante de educación superior es la calidad y cantidad de orientación profesional proporcionada por las instituciones que los albergan, las cuales se traducen en prácticas y experiencias diversas (Reay et al., 2001).

Para estudiar cosas básicas de las matemáticas, como que me perdía un poco, estudiaba de libros y de YouTube, tutoriales... 'el tío Julio', salva mucho. Lo conocí acá, ni siquiera en la media lo veía. Y los libros y más que nada hartos ejercicios (E27).

Jamás había tenido que estudiar tanta materia en mi vida, fue demasiada materia y, claro, acumulé todo para el final, como lo hacía antes, y no me dio el tiempo. No funcionó para nada, intenté cambiar después, acostumbrarse a un hábito es como un poco complicado al principio, porque igual tener 18 años y uno ha tenido toda una vida un hábito de estudiar de forma distinta (E14). 
Los entrevistados advierten que la llegada a la universidad los obliga a movilizar competencias personales orientadas a asegurar su integración social, pues se dan cuenta de que las exigencias de las carreras y el proceso mismo de formación lo requiere. Todos los relatos destacan que este aprendizaje es complejo, los tensiona, implica tiempo, supone ensayo y error, y tomar decisiones. No obstante, en estos mismos relatos, los jóvenes reconocen su capacidad para enfrentar las exigencias y salir adelante a pesar de las dificultades, gestionando sus procesos de aprendizaje.

Integrarme a un grupo fue también caótico, porque al principio es más o menos ensayo y error, hacer el trabajo como con la primera persona que habla, y de a poco uno se va dando cuenta de cómo son las personas. A mí me costó un semestre, trabajar con gente un poco desordenada, que no iba a mi ritmo, que tampoco éramos muy similares en formas de pensar, de organizarnos... ya al otro semestre uno va a eligiendo (E28).

Soy bastante tímido, entonces, al principio, tenía como este temor de acercarme a cualquier persona, pero pensé que como, si ya estaba en la universidad, tenía que hacer un grupo o una red de contactos, así es que me esforcé en hablar con el grupo que encontré, de repente fui cambiando, hasta que llegué a mi núcleo de amistad (E23).

\section{d. Uno se da cuenta del mundo real...}

La permanencia del estudiante egresado de la modalidad técnico-profesional en la universidad se resuelve desde el punto de vista académico y social, con costos (personales y familiares) y beneficios. Los primeros se refieren al deterioro en la calidad de vida y a la pérdida de tiempos personales y familiares. También se expresan en que la continuidad de estudio y el postergar la incorporación al mundo del empleo los deja en posición de desventaja comparativa respectos de sus pares del liceo.

Bueno, alejarme un poco de mi vida social, porque antes estaba en un proceso de tener muchos amigos. La familia igual, no verlos todos los días, el hecho de venirme a vivir [a otro lugar] ya eso es otro costo, y dejar actividades de lado (E12).

Los costos fueron no seguir trabajando, trabajar full-time, generar plata [dinero], quizás ya tendría un subsidio, una casa, poder aportar más en la casa, en cierta manera, tener más riquezas. Mis compañeros del liceo, muchos están trabajando, tienen un auto, tienen una casa, todos tienen título de técnico. Uno se pregunta ¿pero y por qué no lo hice así? (E15).

Por otro lado, se evalúa la perdida de espacios de socialización, debido al tiempo invertido en el traslado al centro educativo y el alto esfuerzo académico para cumplir con la aprobación de asignaturas. Asociada a la idea de costo está la constatación de que todo proyecto supone una complejidad que es preciso reconocer, para empezar a desplegar las acciones que aseguren la permanencia en la carrera.

Mucho estrés, porque cuando uno trabaja o cuando termina el liceo como que vive el día y uno no piensa mucho en lo que viene, pero estando en la universidad el estrés es siempre, porque se aproxima una prueba o un trabajo de investigación (E19).

Uno se da cuenta del mundo real, y eso te tira para abajo. Es un costo también. Uno se da cuenta de que las cosas no son fáciles, pero tampoco pensaba que iban a ser tan difíciles. A pesar de tener el beneficio de la gratuidad, al final eso es un pelo de la cola ${ }^{2}$, pagar o no pagar, ha sido más difícil mantenerse acá. Ha sido duro (E26).

2. Chilenismo para decir "es lo de menos importancia". 
Llama la atención la referencia al tiempo como uno de los costos más sentidos por los estudiantes, en términos de espacio personal para vincularse con otros y del desarrollo de actividades más allá de lo académico. Se interpela la mirada adulto-céntrica del deber ser que deja fuera los aspectos de la vida cotidiana y el desarrollo afectivo-social.

Bueno, me perdí prácticamente la infancia entera de mi hijo porque había momentos en que hacía cosas nuevas y yo nunca estaba. Tuve la suerte de estar en sus primeros pasos, fue pura coincidencia. De mi vida familiar también, uno dedica mucho tiempo a la universidad, entonces, se pierde harto (E29).

Vivo en Quepe, camino a Huichahue, es un sector rural, el cual queda a $5 \mathrm{kms}$. de donde pasa la micro ${ }^{3}$, y debo caminar para tomar la micro que va a la universidad (...) En invierno, con lluvia, con viento, con frío, con heladas, con niebla, con todo. Demoro mucho en llegar a la universidad. Yo creo que entre tres a cuatro horas (E6).

Entre los beneficios, identifican el acceso a mejores oportunidades futuras asociado a la noción de movilidad social, que más allá de los beneficios económicos se representa como la libertad de elegir y el acceso a nuevos estilos de vida. En este escenario, y con base en el conjunto de constricciones sociales y materiales de origen, los jóvenes ven a la educación universitaria como la estrategia para alcanzar el proyecto de vida deseado.

Que voy a tener un trabajo estable, voy a poder optar a darle, quizá, la oportunidad a mi hijo de crecer en un ambiente donde él sepa que va a poder hacer lo que él quiera, ser quién quiera, la seguridad de que va a poder estudiar, poder elegir, quizá lo motive a informarse de ciertas cosas que yo no lo hice cuando empecé a estudiar (E7).

Una visión distinta del mundo; en realidad, con toda esta nueva información que he recibido puedo ayudar a mi familia a contemplar el mundo de otra forma (E28).

\section{Conclusiones}

Hemos presentado los resultados de una investigación que muestra la capacidad de agencia de jóvenes egresados de la modalidad técnico-profesional quienes, sorteando su origen social, ingresan a la educación superior, conviviendo o enfrentando su habitus individual con los habitus institucionales, que pueden facilitar u obstaculizar su integración académica y social.

A partir del análisis de los resultados, se concluye que el acceso a la modalidad técnico-profesional para estos jóvenes está mediado por las condiciones de pobreza y las expectativas del grupo familiar, resultando en una segregación socioeconómica entre modalidades. En ellos, existe una narrativa colectiva respecto de la precariedad de la formación recibida; la vivencia escolar gira en torno a la condición de déficit y falta de expectativas futuras, estableciéndose una noción de riesgo e incertidumbre y, con ello, la búsqueda de un futuro más seguro. No obstante, existe una diversidad de expresiones, dependiendo de la territorialidad y dependencia del establecimiento educativo. Los jóvenes de liceos rurales reconocen una estructura de oportunidades educativas precaria, en la que no existe la posibilidad de elección, debido a una oferta educativa reducida. En tanto, los jóvenes de liceos urbanos se enfrentan a condiciones de alta segregación y mayores niveles de conflictividad en los espacios escolares. Consecuentemente, hay una fuerte resistencia al proyecto curricular de los profesores y sus prácticas institucionales. Esta condición cambia en establecimientos subvencionados confesionales, en los que se valora el apoyo socioafectivo brindado para el desarrollo de proyectos de vida.

3. Forma chilena de denominar al bus de transporte público. 
La valoración de la experiencia técnico-profesional se percibe como compleja y tensa. Por un lado, constituye una imposición para los más pobres y, por otro, representa la oportunidad de obtener credenciales para incorporarse de manera temprana al mundo laboral. Los jóvenes advierten, que, si bien el tracking curricular los excluye de la educación superior, en la actualidad existen diversos espacios de integración, como programas de acceso inclusivo, gratuidad, entre otros, que abren oportunidades a estudiantes con trayectorias educativas exitosas. Sin embargo, se cuestiona la noción de meritocracia que albergan estas políticas públicas, advirtiendo que es excluyente y que se debe transitar hacia un sistema educativo que opere bajo la lógica del reconocimiento, más inclusivo y con oportunidades educativas de calidad para todos. Esto debiera ser posible de subsanar, considerando que en el sistema escolar chileno la educación técnico-profesional compromete una formación orientada al desarrollo de habilidades para continuar realizando estudios, ya sea en el ámbito de la capacitación laboral o en la educación superior técnica o académica.

El tránsito hacia la educación superior reviste un nuevo desafío para estos estudiantes, dado que su integración académica y social en la universidad está teñida por situaciones en las que se presenta discriminación social, falta de preparación académica y de apoyo docente. Durante esta etapa, se evidencia el desarrollo de una conciencia reflexiva respecto de las desigualdades estructurales en las que han estado inmersos en su trayectoria educativa. En este contexto, se plantea el reto a las universidades de acompańar estos procesos, dándoles mayor contenido y sentido formativo, para que estas experiencias se contemplen como espacios para la transformación social, y no de conflicto.

Los jóvenes reconocen que el habitus institucional, expresado en las prácticas organizacionales y personales de los docentes universitarios, es un elemento clave para ampliar las estructuras de oportunidad y dar respuestas sensibles frente a las demandas de integración académica y social. Por lo tanto, se recomienda a las universidades reconocer la especificidad del estudiante de origen técnico-profesional y tomar conciencia de las implicancias que tiene el habitus institucional en estos procesos (Concha, Sánchez, \& Schilling, 2019), con la intención de facilitar los procesos de integración.

Finalmente, la creciente incorporación de egresados de la modalidad técnico-profesional a la educación superior hace imprescindible que, a nivel universitario, se intensifiquen las relaciones de bidireccionalidad con los liceos de enseñanza media para reconocer el perfil del estudiante de esta modalidad y focalizar la pertinencia de los procesos de inducción y nivelación de conductas de entrada, facilitando el tránsito postsecundario de los estudiantes.

El artículo original fue recibido el 3 de enero de 2020

El artículo revisado fue recibido el 3 de noviembre de 2020

El artículo fue aceptado el 7 de enero de 2021

\section{Referencias}

Agencia de Calidad de la Educación. (2016). Panorama de la educación media técnico profesional en Chile. Recuperado de http://archivos.agenciaeducacion.cl/Panorama_Ed_TP_en_Chile.pdf

Archer, L. \& Hutchings, M. (2000) Bettering Yourself? Discourses of risk, cost and benefit in ethnically diverse, young working-class non-participants constructions of higher education. British Journal of Sociology of Education, 21(4), 555-574. https://doi.org/10.1080/713655373

Aisenson, D. (2002). Después de la escuela. Transición, construcción de proyectos, trayectoria e identidad de los jóvenes. Buenos Aires, Argentina. EUDEBA

Ainsworth, J. W. \& Roscigno, J.W. (2005). Stratification, School-Work Linkages and Vocational Education. Social Forces, 84(1), 257-284. https://doi.org/10.1353/sof.2005.0087 
Arias, E., Farías, M., González-Velosa, C., Huneeus, C., y Rucci, G. (2015). Educación Técnico Profesional en Chile. Recuperado de https://publications.iadb.org/publications/spanish/document/Educaci\%C3\%B3nt\%C3\%A9cnico-profesional-en-Chile.pdf

Arum, R. \& Shavit, Y. (1995). Secondary vocational education and the transition from school to work. Sociology of Education, 68(3), 187-204. https://doi.org/10.2307/2112684

Ashworth, P. D. (2000). Métodos cualitativos de investigación. Estudios pedagógicos (Valdivia), (26), 91-106. https://doi.org/10.4067/S0718-07052000000100007

Bassi, M. y Urzúa, S. (2010). Educación en Chile: el desafío está en la calidad. Recuperado de https://publications.iadb.org/ publications/spanish/document/Educaci\%C3\%B3n_en_Chile_El_desaf\%C3\%ADo_est\%C3\%A1_en_la_calidad.pdf

Bellei, C. (2013). El estudio de la segregación socioeconómica y académica de la educación chilena. Estudios pedagógicos (Valdivia), 39(1), 325-345. https://doi.org/10.4067/S0718-07052013000100019

Bourdieu, P. (1980). El sentido práctico. Madrid, España: Taurus

Bourdieu, P. \& Wacquant, L. (1992). An Invitation to Reflexive Sociology. Chicago, IL: The University of Chicago Press.

Bourdieu, P. y Passeron, J. C. (1995). La Reproducción: elementos para una teoría del sistema de enseñanza. Barcelona, España: Fontamara.

Bonilla-García, M. y López-Suárez, A. (2016). Ejemplificación del proceso metodológico de la teoría fundamentada. Cinta de Moebio, Revista de Epistemología de las Ciencias Sociales, (57), 305-315. https://doi.org/10.4067/S0717-554X2016000300006

Canales, M. (2006). Metodologías de investigación, introducción a los oficios. Santiago, Chile: LOM.

Canales, A. y De los Ríos, D. (2009). Retención de estudiantes vulnerables en la educación universitaria. Calidad en la Educación, (30), 50-83. https://doi.org/10.31619/caledu.n30.173

Canales, M., Opazo, A., y Camps, J. (2016). Salir del cuarto: Expectativas juveniles en el Chile de hoy. Revista Última década, 24(44), 73-108. https://doi.org/10.4067/S0718-22362016000100004

Castillo, J. y Cabezas, G. (2010). Caracterización de jóvenes primera generación en educación superior. Nuevas trayectorias hacia la equidad educativa. Calidad en la Educación, (32), 44-76. https://doi.org/10.31619/caledu.n32.151

Catalán, X. (2016). Elección de modalidad educativa en la Enseñanza Media y su rol en la postulación a las universidades del CRUCH. Calidad en la Educación, (45), 288-320. https://doi.org/10.4067/S0718-45652016000200009

Concha, C. (2009). Sujetos rurales que por primera generación acceden a la universidad y su dinámica de movilidad social en la Región del Maule. Calidad en la Educación, (30), 122-158. https://doi.org/10.31619/caledu.n30.175

Concha, C., Sánchez, G., \& Schilling, C. (2019). Acoplamiento entre habitus institucional y habitus individual: una lectura desde la experiencia de estudiantes de enseñanza media Técnico profesional que estudian en tres universidades regionales del CRUCH. (Vol.N¹4). Cuadernos de Investigación en Aseguramiento de la Calidad, (14). Recuperado de https://investigacion.cnachile.cl/archivos/cna/documentos/Cuaderno-14_C.Concha.pdf

Crozier, G., Reay, D., Clayton, J., Colliander, L., \& Grinstead, J. (2008). Different strokes for different folks: diverse students in diverse institutions - experiences of higher education. Research Papers in Education, 23(2), 167-177. https://doi.org/10.1080/02671520802048703

Dávila, O., Ghiardo, F., \& Medrano, C. (2005). Los desheredados. Valparaíso, Chile: CIDPA.

de Iruarrizaga, F. (2009). Dos miradas a la educación media en Chile. Santiago, Chile: Instituto de Economía de la Pontificia Universidad Católica de Chile.

Dumay, X. \& Dupriez, V. (2008). Does the school composition effect matter? Evidence from Belgian data. British Journal of Educational Studies, 56(4), 440-477. https://doi.org/10.1111/j.1467-8527.2008.00418.x

Farías, M. y Carrasco, R. (2012). Diferencias en resultados académicos entre educación técnico-profesional y humanistacientífica en Chile. Calidad en la Educación, (36), 87-121. https://doi.org/10.31619/caledu.n36.118

Flick, U. (2004). Introducción a la Investigación Cualitativa. Madrid. España: Ediciones Morata S. L.

Giroux, H. (1985). Teorías de la reproducción y la resistencia en la nueva sociología de la educación: un análisis crítico. Cuadernos Politicos, (44), 36-65. Recuperado de http://www.cuadernospoliticos.unam.mx/cuadernos/ contenido/CP.44/cp.44.6.\%20HenryAGiroux.pdf 
Guba, E. y Lincoln, Y. (2002). Paradigmas en competencia en la investigación cualitativa. En C. Derman \& J. Haro (Eds.), Por los rincones. Antología de métodos cualitativos en la investigación social (pp. 113-145). Sonora, México: El Colegio Sonora.

Hirsch, D. \& Rio, V. (2015). Teorías de la reproducción y teorías de la resistencia: una revisión del debate pedagógico desde la perspectiva materialista. Foro de Educación, 13(18), 69-91. https://doi.org/10.14516/fde.2015.013.018.004

Jenkins, S. P., Micklewright J., \& Schnepf, S. V. (2008). Social segregation in secondary schools: how does England compare with other countries? Oxford Review of Education, 34(1), 21-37. https://doi.org/10.1080/03054980701542039

Larrañaga, O., Cabezas, G., y Dussaillant, F. (2013). Informe completo del Estudio de la Educación Técnico Profesional. Recuperado de http://www.cl.undp.org/content/dam/chile/docs/pobreza/undp_cl_pobreza_ETP_2013.pdf

Larrañaga, O., Cabezas, G., y Dussaillant, F. (2014). Trayectorias educacionales e inserción laboral en la enseñanza media técnico-profesional. Estudios Públicos, (134), 7-58. Recuperado https://www.cepchile.cl/cep/site/ docs/20160622/20160622111507/revista_estudios_publicos_134.pdf

Mauna, M. (2013). Una aproximación cualitativa al estudiante de primera generación en la educación superior chilena. En M. E. Irigoin, R. del Valle, \& C. Ayala (Eds.), Acceso y Permanencia en la Educación Superior. Sin apoyo no hay oportunidad (pp.15-51). Santiago, Chile: Foro Aequalis.

McDonough, P. (1988). Classmates? How students prepare for different college futures. Trabajo presentado en el Encuentro anual de la American Educational Research Association. New Orleans.

Mejía-Pérez, G. y Worthman, S. S. (2017). La geografía de las oportunidades. El caso de las sedes de las universidades autónomas en municipios con poca oferta de educación superior. Revista Iberoamericana de Educación Superior, 8(23), 25-48. https://doi.org/10.22201/iisue.20072872e.2017.23.244

Ministerio de Educación. (2020). Estudio sobre trayectorias educativas y laborales de estudiantes de educación media técnico-profesional. Evidencias, 46. Santiago, Chile. Recuperado de https://centroestudios.mineduc.cl/ wp-content/uploads/sites/100/2020/04/EVIDENCIAS-46_2020_f02.pdf

Ong, P. \& Rickles, J. (2004). The Continued Nexus between School and Residential Segregation. La Raza LJ, 15(1). https://doi.org/10.15779/Z38ZH3M

Organización para la Cooperación y el Desarrollo Económicos (OCDE). (2015). Estudio económico de la OCDE en Chile (archivo PDF). Recuperado de https://www.oecd.org/eco/surveys/chile-2015-volante.pdf

Orfield, G. (2001). Schools more separate: Consequences of a decade of resegregation. Cambridge, MA: Harvard University Press.

Programa de las Naciones Unidas para el Desarrrollo (PNUD). (2017). Desiguales. Origenes, cambios y desafíos de la brecha social en Chile. Santiago, Chile: Programa de las Naciones Unidas para el Desarrollo.

Raczynski, D., Hernández, M. Rejevic, L., y Roco, R. (2011). El paso de la enseñanza básica a la media en estratos bajos: un reto a la igualdad de oportunidades. Recuperado de https://centroestudios.mineduc.cl/wp-content/uploads/ sites/100/2017/07/Informe-Final-Dagmar-Raczynski-F511083.pdf

Reay, D., David, M., \& Ball, S. (2001). 'Making a Difference?: Institutional Habituses and Higher Education Choice. Sociological Research Online, 5(4), 126-142. https://doi.org/10.5153/sro.548

Reay, D. (1998). 'Always knowing' and 'never being sure': familial and institutional habituses and higher education choice. Journal of Education Policy, 13(4), 519-529. https://doi.org/10.1080/0268093980130405

Sevilla, M. P. \& Polesel, J. (2020). Vocational education and social inequalities in within-and between-school curriculum tracking. Compare: A Journal of Comparative and International Education. https://doi.org/10.1080/03057925.2020.1798214

Sevilla, M. (2017) Especialización vs Polivalencia en la educación media. ¿Es relevante el tipo de tracking curricular en la segregación escolar y el acceso a la educación superior? Cuadernos de Educación, (77). Recuperado de http://repositorio.uahurtado.cl/handle/11242/10503

Shavit, Y. \& Müller W. (2000). Vocational Secondary Education, Tracking, and Social Stratification. En M. T. Hallinan (Ed.), Handbook of the Sociology of Education (pp. 437-452). Boston, MA: Springer. https://doi.org/10.1007/0-387-36424-2_20

Treviño, E., Valdés, H., Castro, M., Costilla, R., Pardo, C., \& Donoso, F. (2010). Factores asociados al logro cognitivo de los estudiantes de América Latina y El Caribe. Santiago, Chile: UNESCO. Recuperado de https://unesdoc.unesco.org/ark:/48223/pf0000186769 
Valenzuela, J. P., Bellei, C., y De los Ríos, D. (2009). Evolución de la segregación socieconómica de los estudiantes chilenos y su relación con el financiamiento compartido. En Fondo de Investigación y Desarrollo (Ed.), Evidencias para Políticas Públicas en Educación (pp.231-284). Santiago, Chile: Ministerio de Educación.

Zimmer, R. \& Toma, E. (2000). Peer effects in private and public schools across countries. Journal of Policy Analysis and Management, 19(1),75-92.https://doi.org/10.1002/(SICI)1520-6688(200024)19:1<75::AID-PAM5>3.0.CO;2-W 